\title{
Power Management and Energy Storage Experiments on a MW-Scale Naval Power System Test-bed
}

\author{
S. Pish, J. Herbst, D. Wardell, A. Gattozzi, M. Flynn \\ University of Texas Center for Electromechanics \\ Austin, Texas \\ s.pish@cem.utexas.edu
}

\begin{abstract}
From local utility grids to electric ships, providing power to an ever increasing variety and magnitude of loads requires power grids that are more flexible, resilient, and efficient than ever before. In many cases, these systems must serve loads that are nearly equal to and occasionally exceed peak generation capacity. As a result, it is critical that these power systems and their controls are analyzed with high-fidelity models validated through component and system level testing. The University of Texas' megawatt scale MVDC/HFAC power system testbed supports Navy programs and other research on isolated power systems. Testing completed to date includes model validation of a high fidelity model of series dc arc faults, investigation of rapid power transfer among multiple loads and sources, and coordination of energy storage. This paper presents the development of a power system test bed along with relevant research findings. In addition, key issues related to power management including energy storage and system inertial response are addressed.
\end{abstract}

Keywords-Microgrid, power, controls, MVDC, modeling, energy

\section{INTRODUCTION}

Emerging electric loads and the need to maximize resiliency, efficiency and performance of future naval platforms drive the US Navy towards integrated electric power systems (IPS). At power levels approaching $100 \mathrm{MW}$, future IPS architectures will rival the scale of large land-based facilities but will likely be required to fit within hulls of 7,000 - 10,000 ton displacement to be practical and affordable. Modeling and simulation will play a key role in developing and evaluating the performance of future naval power system architectures.

Component and system level testing support technology development through validation of models used to guide the designs and demonstrating the performance of critical elements of the system. Modeling combined with system/subsystem level testing allows the designer to observe and capture complex interactions among the system components and the control system -- including instrumentation, sensors and communication networks -- that are extremely difficult to accurately model in advance. Validated models can then be used to project the performance of the system under different conditions and at different scales.

The University of Texas created a flexible, megawatt-scale electric power system test bed, shown in Figure 1, along with high fidelity simulation models of the components and controls $[1,2,3]$. In addition to model validation and demonstration of critical performance characteristics of future naval power systems, the test-bed is used to conduct power- and control hardware-in-the loop-testing to develop and refine new control algorithms and evaluate fault mitigation and protection approaches. A description of the system used for the recent MVDC testing conducted under an ONR sponsored program as well as the objective configuration for the facility after completion of ongoing upgrades will be presented.

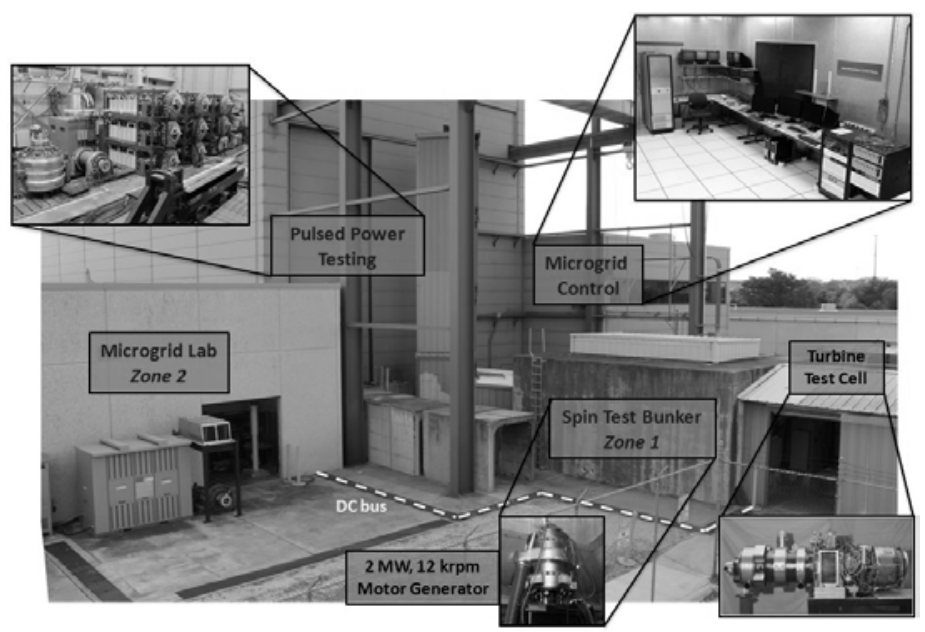

Fig. 1. General arrangement and key components of the UT distributed power system facility.

\section{TEST-BED DESCRIPTION}

The objective configuration of the UT power system test-bed is shown in Figure 2; shaded items are being installed as part of the facility upgrade. The test-bed is currently configured to represent a notional MVDC ship power system with a $1,150 \quad \mathrm{~V}_{\mathrm{dc}}$ distribution voltage. The test-bed features a $50 \mathrm{~m}$ distributed multi-conductor bus connecting two discrete lab spaces. Power for the test-bed can be supplied from the facility's electric distribution grid or from isolated diesel or gas turbine driven generators. A previous study investigated series arc faults in dc systems for the development of realistic models of those faults [4]. Recent experiments featured a distributed main bus with two distribution zones, two independent power sources, and both steady-state and transient loads. 
The following hardware was installed and operational for use during testing of the objective UT naval power system.

- Motor/generator, 2 MVA, 12,000 rpm

- 5 MW dynamometer (Kahn)

- $\quad$ 1.3 MW Resistive load and 2 MW chopper

- $\quad$ Rectifiers: 3.2 MVA (diode), 1.2 MVA (controlled), 1 MVA (Toshiba)

- Inverters: 1 MVA (Toshiba), 2 MVA (ARCP)

- Utility Power: two 480 Vac $3 \varphi$ utility supplies

- $\quad$ Transformers: 490 kVA, 1.2 MVA multi-tap

The test-bed is currently being upgraded with the installation of 10 Semikron Semistack RE converter modules. The Semikron modules are IGBT-based power assemblies designed for configuration as 3-phase inverters or 4-quadrant, 3-phase bidirectional converters. The water-cooled modules are designed for flexible integration and are rated at 1.6 MVA at 1,250 $\mathrm{V}_{\mathrm{dc}}$ and 1,400 A.

Control flexibility is a critical feature of the new power converters and the onboard controllers are being configured to provide complete control over the module control algorithms and enable reconfiguration of the modules for multiple functions.

The NI-based control platform integrates a real-time processor, a user-configurable FPGA, and a full set of power electronics control and communication I/O. Simulation models developed will run on the FPGA board with clock speeds up to $40 \mathrm{MHz}$, enabling real-time emulation of power system components (e.g. energy storage or alternative power generation). Real time digital simulation and the Semikron power modules will be used to perform hardware-in-the-loop (HIL) testing in one of its several variants, e.g. control- or power-hardware-in-the-loop (CHIL or PHIL) at MW power levels, expanding considerably the range of experiments possible on the UT microgrid.

In addition to the enhancements to the microgrid power system, the UT laboratory now also includes a $12 \mathrm{MJ}$ capacitor-based pulse forming network (PFN). The charging power supplies and capacitor banks can be interfaced with the naval power system test-bed to study integration and control of emerging pulsed loads onto isolated power systems. This unique combination of capabilities and expertise in development and demonstration of advanced power systems and pulsed power technologies enable world-class research in advanced naval power system technologies and control systems.

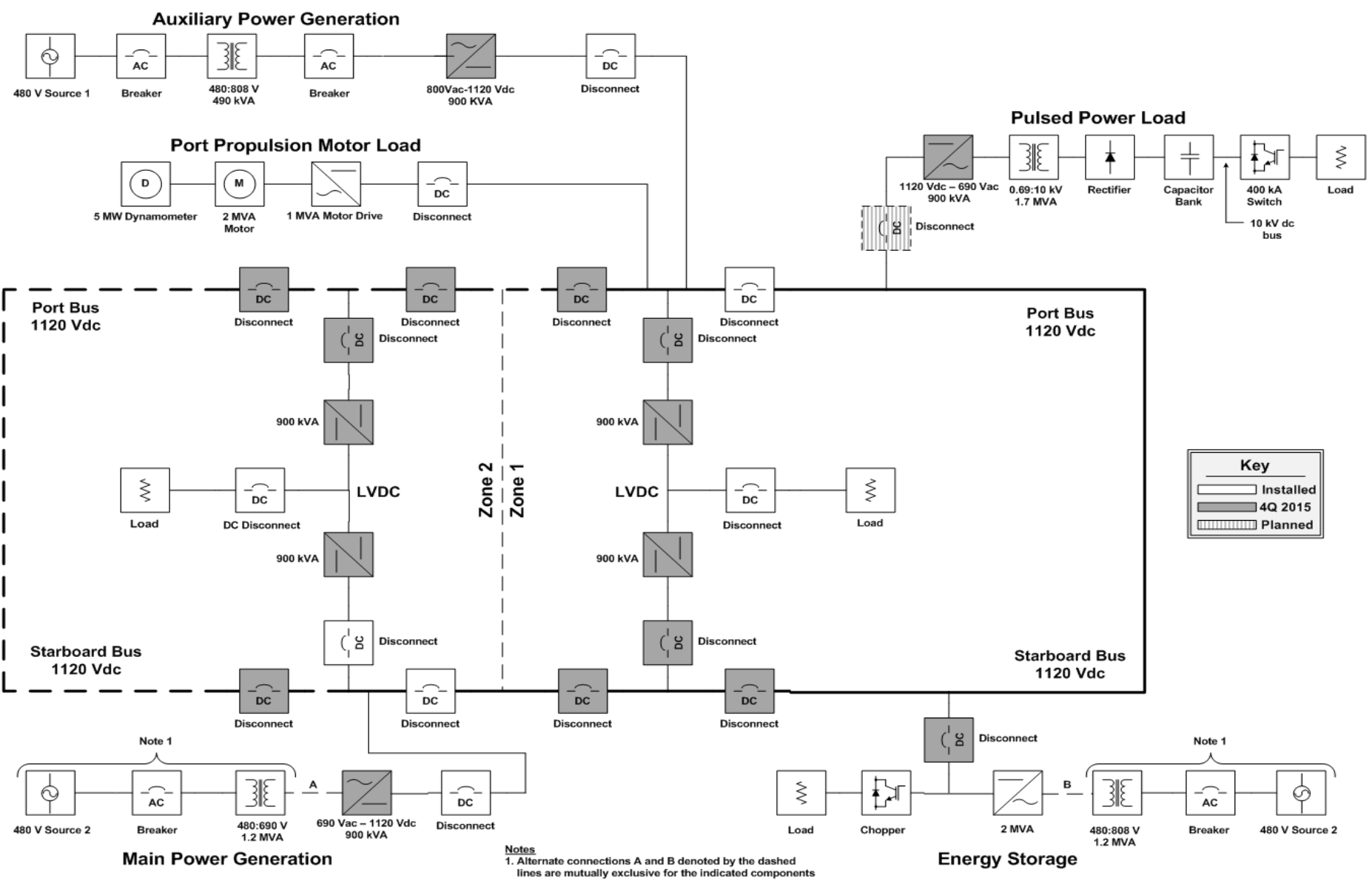

Fig. 2. Diagram of the objective UT naval power system test-bed. 


\section{SimULATION AND TESTING}

Supported by high fidelity simulation models, four experimental demonstrations were conducted: single source load transfer, two source load transfer, source transfer with variable load, and energy storage emulation. Each experiment was conducted once using utility power supplies and later repeated with isolated 300 $\mathrm{kW}$ diesel generators.

A key goal of the program was to demonstrate that well-validated component models could be assembled into system-level models that were capable of accurately predicting the system-level behavior under a variety of test conditions. Accordingly, comparisons of predicted versus measured results were based on the original system-level models without taking advantage of "inprocess" refinements to improve model/test correlation. Minor differences between predicted and actual behavior were in fact observed during the program. These differences illustrate the challenges of accurately representing all relevant system parameters (in this case a slight difference between calculated and actual bus impedance) and demonstrate the value of hardware testing to understand the source of observed anomalies and identify refinements to the models.

\section{A. Power system modelling}

Detailed component and system-level models of the physical hardware were completed and exercised before the experimental activities. The system-level models were used to develop control strategies and predict the behavior of the system so that experiments could be conducted safely on physical hardware.

After initial characterization of the individual components, a Simulink model of its microgrid set up, shown in Figure 3, was developed to guide the test program. The model consists of four major sections which are each comprised of additional blocks and sub-blocks. For example, the Auxiliary Power Generation (APG) section includes a Turbine and Alternator block, shown in Figure 4, and AC-DC Rectifier block. Models were re-configured as needed between experiments to reflect changes in the setup of physical hardware for each test sequence.

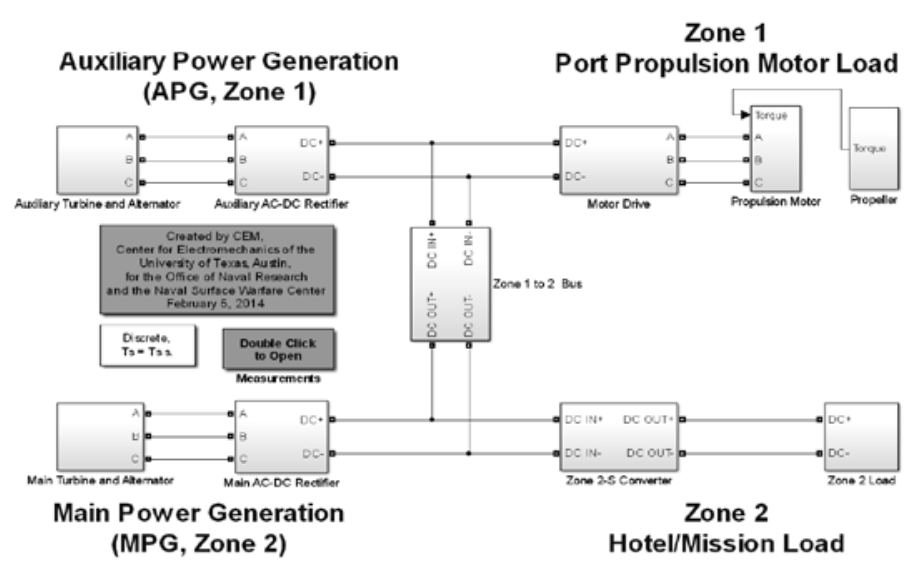

Fig. 3. UT Simulink model of the naval power system test-bed.

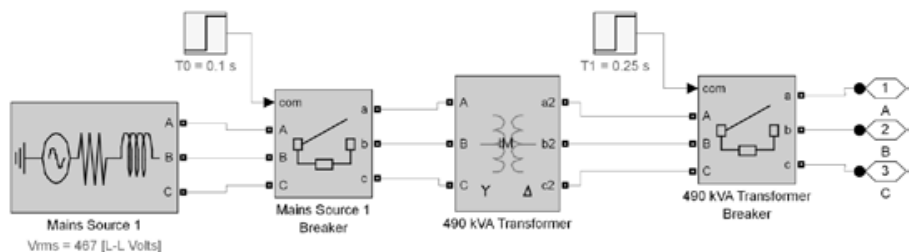

Fig. 4. Internal structure of the UT Simulink modelTurbine and Alternator subblock using an ideal voltage source.

In developing the simulation model, standard Simulink and SimPowerSystems blocks were used to represent the system components. These have sufficient fidelity to accurately model relevant system dynamics. Salient modeling simplifications were made when appropriate. For example, control loop delays were ignored when small compared to the time constants of the major system components. In addition, because it was not the focus of the test effort, the dynamometer was modelled as a simple torque command rather than as a detailed hydro-mechanical system.

\section{B. Testing}

The goals of the four experiments were to demonstrate and evaluate the controls of the power system test bed, examine the system's reaction to load and source transfer events, and verify model predictions.

The system controls required to modulate one load in reaction to other transient loads were demonstrated and the overall system response to the perturbations was minimal. This is an important aspect of the experiment and control scheme because maintaining a steady voltage on the dc bus at the prime power output helps maintain ship power quality and stability while allowing prime power generation to operate at improved efficiency.

\section{1) Single-source load transfer}

The first power transfer experiment demonstrated rapid load transfer between two loads of comparable scale with a single passive rectifier as the power source for the dc distribution bus. This test sequence represents a scenario where a major load such as propulsion is modulated in response to rapid load changes of significant scale (relative to the base loading) on the dc bus. Total power was kept at $500 \mathrm{~kW}$ and is shown in the power vs time plot in Figure 5.

\section{2) Two-source load transfer}

The two-source load-switching test demonstrated the controlled transfer of power between two ship loads and two varying sources while maintaining the aggregate source power near 500 $\mathrm{kW}$. As noted above and shown in Figure 6, minor differences between the predicted and experimental results were observed during the two source load transfer experiment. In this case, the actual bus impedance was higher than the calculated value used in the model, leading to differences in load sharing between the two sources. 

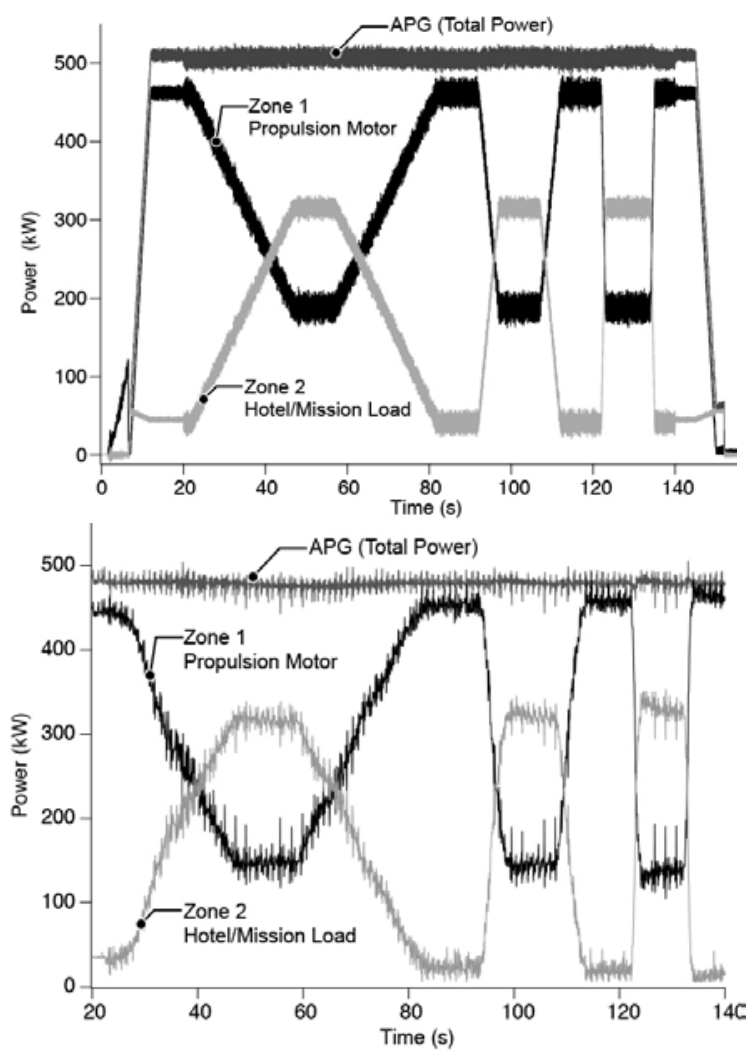

Fig. 5. Single source load transfer model (top) and test data (bottom).
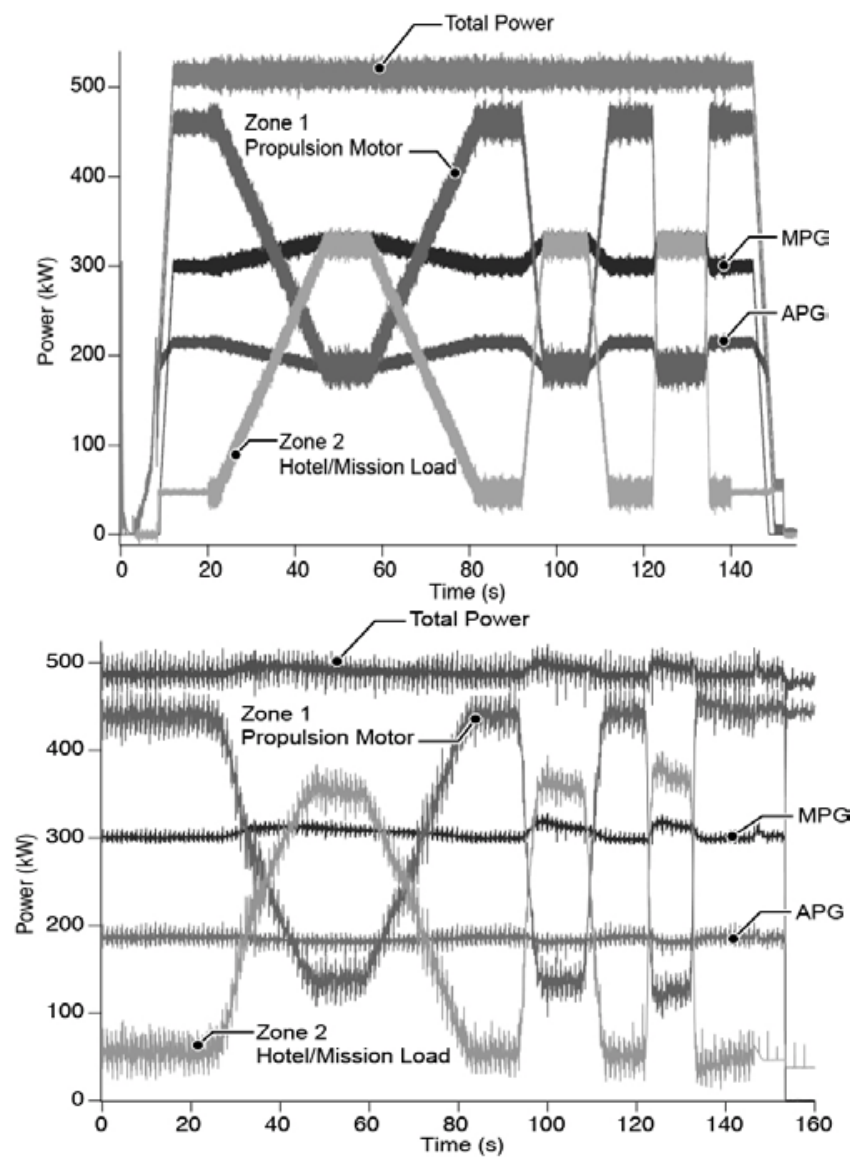

Fig. 6. Two source load transfer model (top) and test data (bottom).

\section{3) Source Transfer}

The source transfer test demonstrated the controlled transfer of power between two ship sources in response to a varying load. This experiment represents a scenario where the ship transitions between sources to maintain peak efficiency and provide additional power generation as load varies. For this experiment, the dynamometer established a base load of $100 \mathrm{~kW}$ on the dc bus, supplied by one power source. The load was then ramped to $450 \mathrm{~kW}$ and then back down to $100 \mathrm{~kW}$ for the test sequence, as shown in Figure 7. As the load increased, the control system ramped the first power source off and the second power source on. Once the dynamometer exceeded $400 \mathrm{~kW}$, the first source was turned back on to share power. This sequence was then reversed as the dynamometer was ramped from $400 \mathrm{~kW}$ back to the base load.
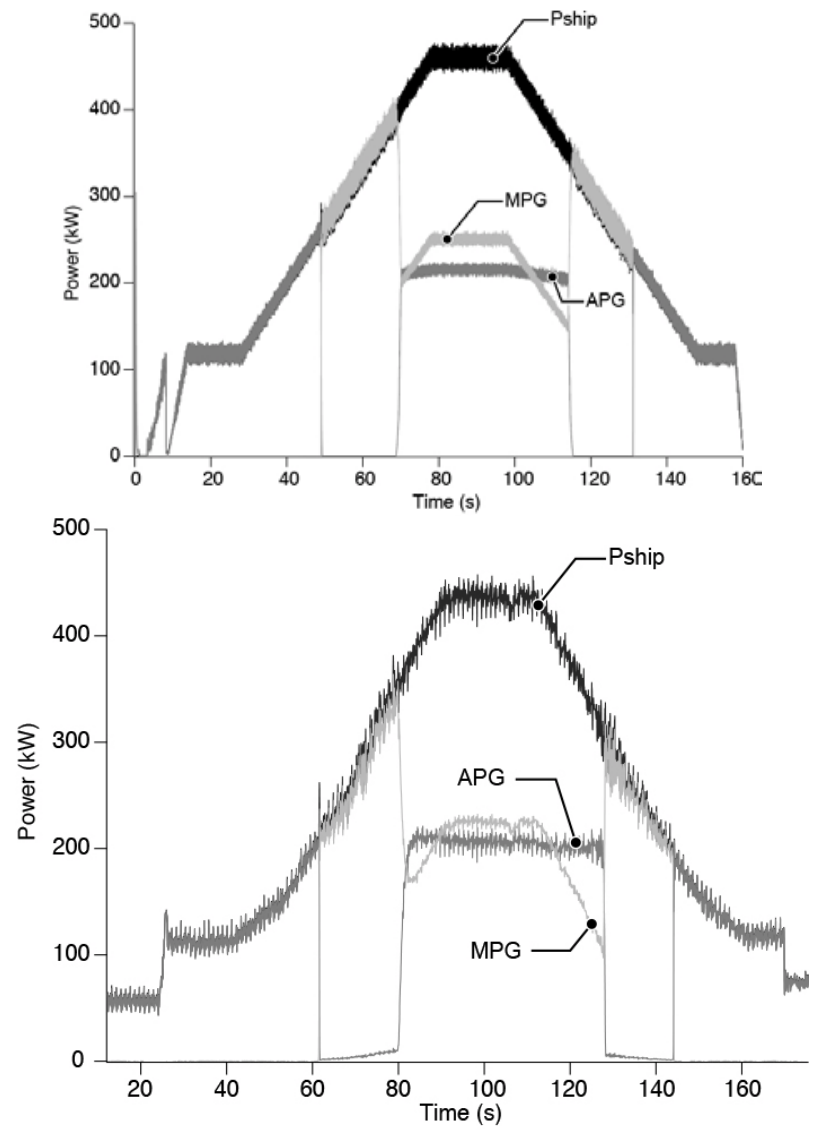

Fig. 7. Source transfer with variable load model (top) and test data (bottom).

\section{4) Energy Storage Emulation}

The energy storage emulation test, shown in Figure 8, demonstrated the service of a large repetitive pulse load while maintaining essentially constant load on the main distribution bus. The pulsed load, notionally a capacitor-based Pulse Forming Network (PFN), operates at a power level that exceeds peak ship generation. This represents a scenario where the ship serves a large repetitive pulsed power load and an energy storage element is charged to maintain ship power constant and then discharged to provide supplemental power to the pulsed load. 

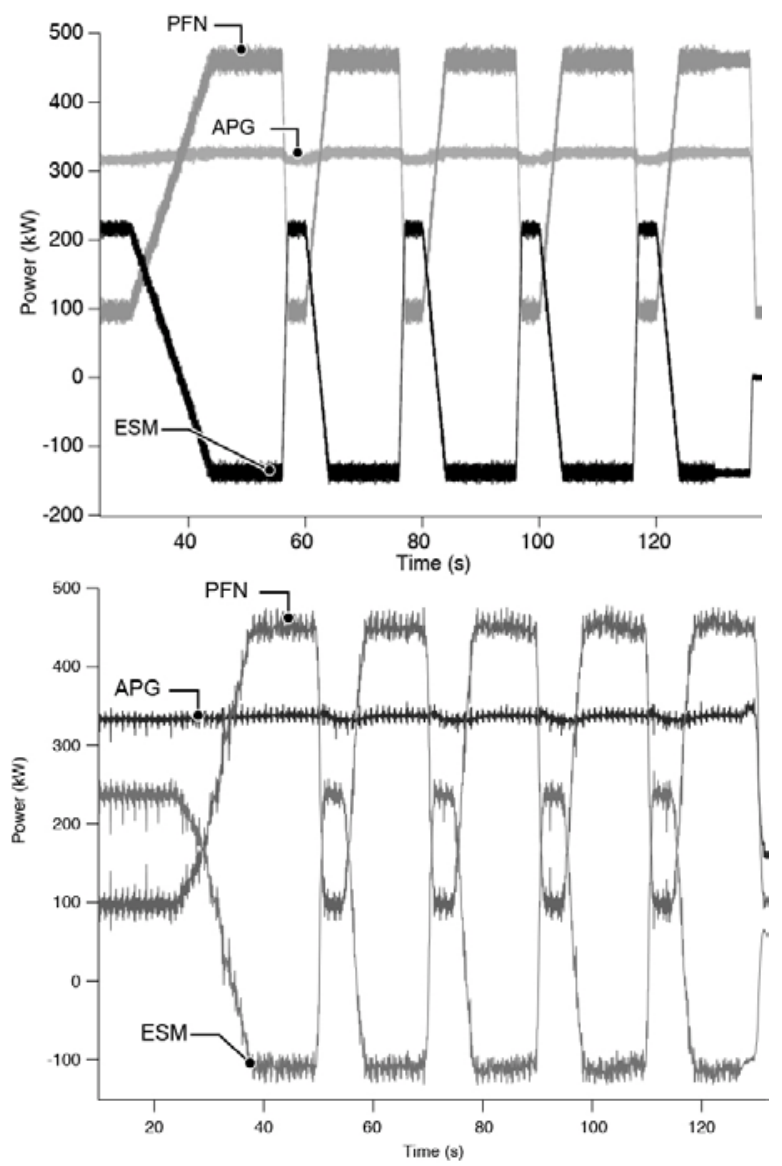

Fig. 8. Energy storage emulation model (top) and test data (bottom).

For this experiment, ship power generation is represented by the Auxiliary Power Generation source held constant near $300 \mathrm{~kW}$. The pulsed load PFN profile is provided by the induction motor driven dynamometer. Energy storage is emulated in two parts. The Energy Storage source uses the same source used in previous experiments as the Port Propulsion Motor Load. The other half of the emulation, or Energy Storage sink, is represented by a chopper and resistor bank.

\section{Soft Source Testing}

After successful completion of the initial four test sequences with the utility power supply, the experiments were repeated using two $300 \mathrm{~kW}$ diesel generator sets to explore the impact of "soft" power sources. For the single and two source load transfer, the power levels were lowered slightly because of the ratings of the available diesel generators. Again, the initial models were used for comparisons between predicted system-level behavior and experimental results without the benefit of subsequent refinements. With the exception of minor variations in the dynamometer response (later shown to be related to operation at the extreme low end of its power capability) the experiments with the diesel generators closely matched the performance predicted by the models as can be seen in Figure 9 .
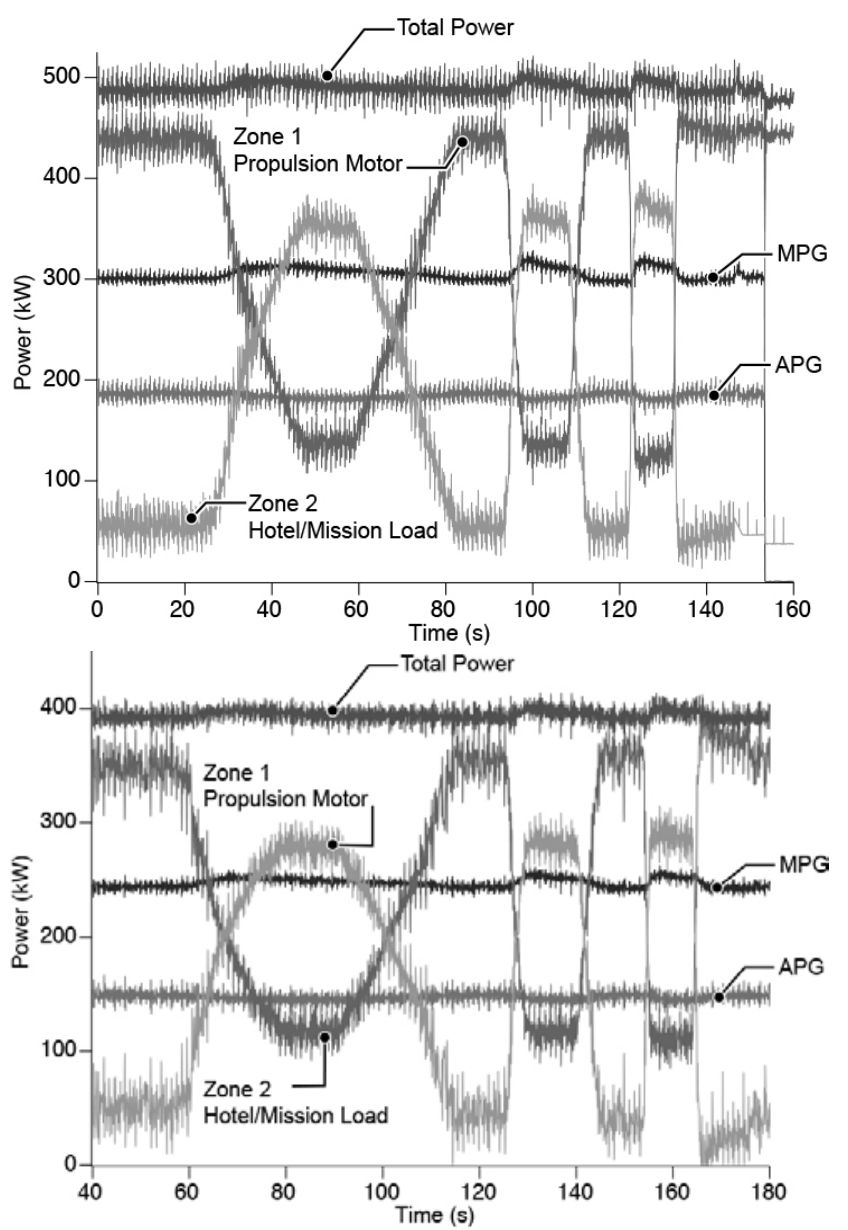

Fig. 9. Test data from two-source load transfer tests with utility sources (top) and diesel generators (bottom).

\section{System "Inertial” Response Study}

To capture the response of the engine and generator controllers to transient loading, the diesel generators were tested under a series of step loads, ranging from $110 \mathrm{~kW}$ to $180 \mathrm{~kW}$. As can be seen from Figure 10, the voltage response of the generators is different even though the units are identical in specification- highlighting the need to characterize the actual physical hardware.

The frequency and voltage response of generator 1 to the step loads is shown in Figure 11. Prior to response from the controllers, the system response is dictated by the physical inertia of the engine/generator. At later times in the transient, the response of the engine governor and generator exciter can be observed. These results indicate that exciter response is more critical than governor response. Active rectification can potentially regulate voltage faster than generator exciter response but this warrants further study. Dc distribution systems may be able to accommodate larger frequency excursions on the ac side than legacy ac systems and standards may need to evolve, particularly in dc systems, to reflect new capabilities with modern controls. 

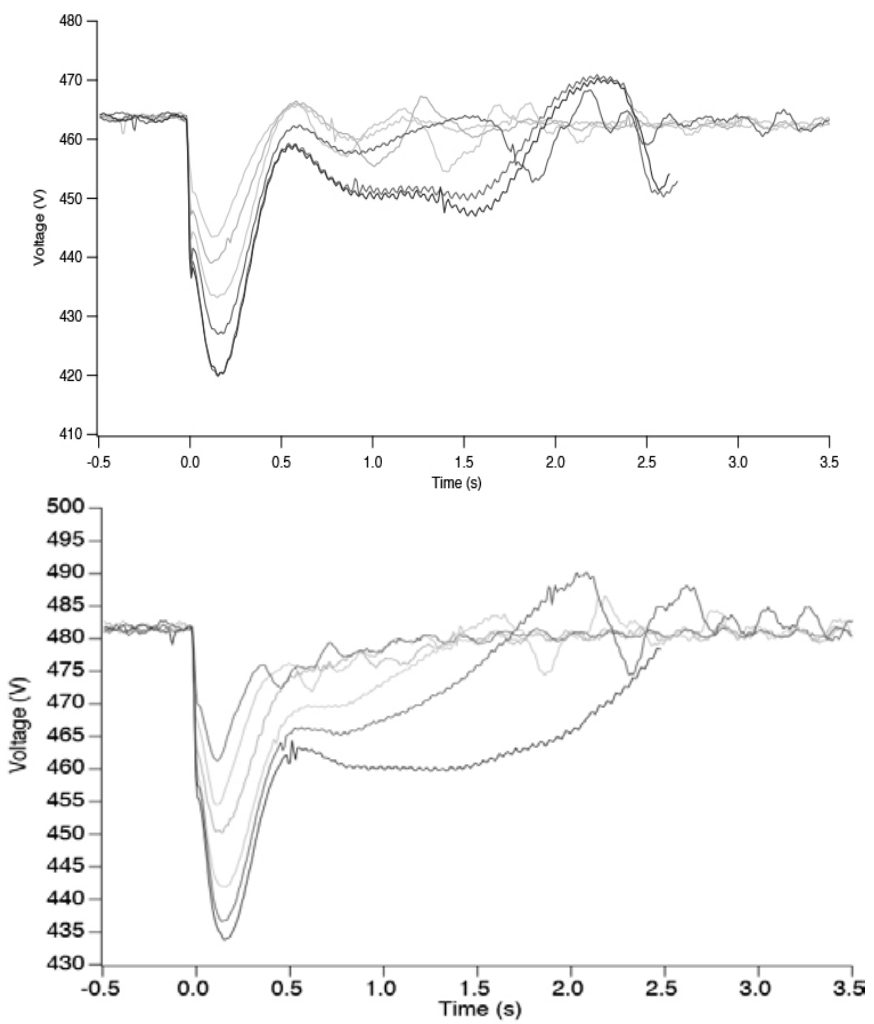

Fig. 10. Step load test data from two identical diesel generators.
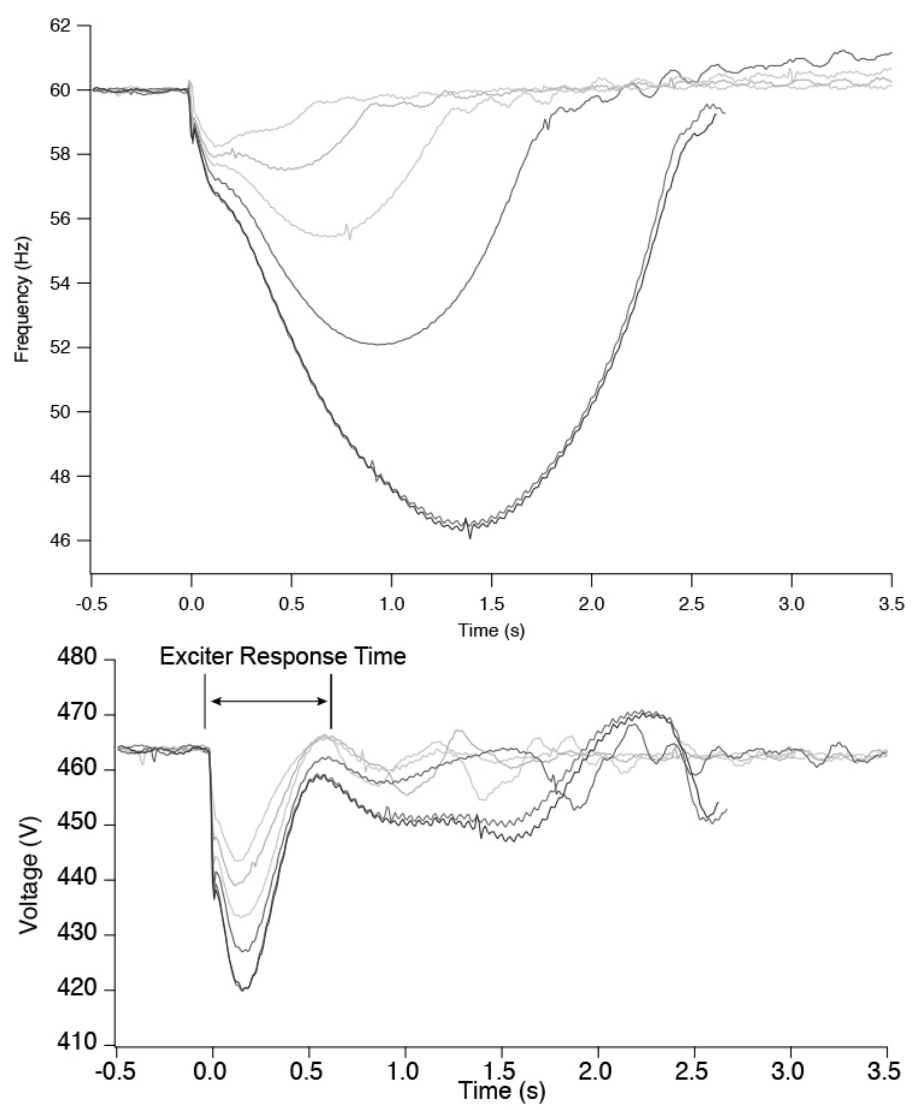

Fig. 11. Generator 1 frequency (top) and voltage (bottom) response to block loading.

\section{CONCLUSIONS}

The flexibility of aspects of potential dc electric ship architectures has been demonstrated at interesting power levels. The experiments validated high-fidelity models of relevant electrical ship architectures. The experiments demonstrate the importance of testing of real systems (with physically separated components, control latency, etc.) to validate models.

There are two major conclusions from this work. The first is that system modeling can be used with confidence, in certain applications, to predict the behavior of isolated power systems in which sources and loads are dynamically and significantly increased or decreased in power. In addition, energy storage can be effectively utilized to stabilize bus voltage and power while serving large transient loads. The experimental validation provides additional confidence in extending this modeling approach to more complex applications.

More importantly, it shows that large scale shifts in power can be handled by today's equipment. This gives confidence that new concepts in ship power specification and control can support large transient loads as well as adding or removing generators for efficiency. Even though the generators had their own safety control system, the system controller could work within the generator constraints to do switching of power levels that constituted a large fraction (occasionally greater than 1) of the available power from the generators. This result bodes well for robust design of future ships.

\section{Acknowledgment}

The authors would like to thank the Office of Naval Research for their guidance and financial support of the test program and converter module upgrade.

\section{REFERENCES}

[1] R. Hebner et al., "Intelligent microgrid demonstrator," in Proc. ASNE Elec. Mach. Technol. Symp., Philadelphia, PA, 2010.

[2] J. D. Herbst et al., "Flexible test bed for MVDC and HFAC electric ship power system architectures for navy ships," in Proc. Elec. Ship Technol. Symp., Alexandria, VA, 2011.

[3] R.Hebner, J.Herbst, andA.Gattozzi, "Large scale simulations of a shippower system with energy storage and multiple directed energy loads," in Proc. Grand Challenges Model. Simul. (GCMS 2010), Ottawa, ON,Canada, pp. 430-435.

[4] F. M. Uriarte, A. L. Gattozzi, J. D. Herbst, H. B. Estes, T. J. Hotz, A. Kwasinski, and R. E. Hebner, "A DC Arc Model for Series Faults in Low Voltage Microgrids”, IEEE Transactions on Smart Grids, Vol. 3, No. 4, December 2012. 